\title{
Desafios Terapêuticos da Insuficiência Coronariana Obstrutiva Crônica
}

\author{
Whady A. Hueb, José Antonio F. Ramires
}

São Paulo, SP

As opções terapêuticas para portadores de angina estável na doença coronariana obstrutiva crônica permanecem conflitantes, motivando grandes debates clínicos. Estudos iniciais de seguimento clínico de pacientes com angina estável demonstraram que o percentual de mortalidade aumentou linearmente com o aumento do número de artérias comprometidas ${ }^{1,2}$. Estudo ulterior, realizado retrospectivamente na Duke University ${ }^{3}$, em 1.214 pacientes, demonstrou incidência de morte anual de 7,2\% com comprometimento proximal nos três ramos coronarianos principais. A comparação desses dados com subgrupos de pacientes, com mesmo número de artérias comprometidas, porém com severa disfunção ventricular, mostrou significativo aumento na incidência de morte. Por outro lado, a relativa baixa mortalidade nos pacientes com função ventricular preservada foi marcante, considerando que nesse subgrupo foi incluída angina instável e excluídos os pacientes livres de angina. De fato, estudos realizados em nosso meio $^{4}$, em 150 pacientes com angina estável e função ventricular preservada, mostraram ausência de mortalidade em portadores de doença uni e biarterial e 1,5\% de mortalidade anual naqueles com estenoses triarterial e tronco da artéria coronária esquerda, após seguimento de até 8 anos (fig. 1). Nesse estudo, foi identificada significativa remissão dos sintomas anginosos atribuída, possivelmente, à abordagem terapêutica maciça, presença de circulação colateral ou até pela oclusão arterial com ou sem manifestação clínica. Além disso, ainda que tenham surgido novas estenoses em segmentos arteriais aparentemente normais, observou-se relativa estabilidade do grau de estenoses prévias e os surgimentos de oclusões arteriais com circulação colateral tiveram implicações definitivas no bom prognóstico dos pacientes.

Instituto do Coração do Hospital das Clínicas - FMUSP

Correspondência: Whady A. Hueb - InCor - Av. Dr. Enéas C. Aguiar, 44 - 05403-000

São Paulo, SP - E-mail: cor_whady@incor.usp.br

Recebido para publicação em 26/2/02

Aceito em 01/07/02
Assim, concluiu-se que os portadores de angina estável com função ventricular preservada, independentemente do número de artérias comprometidas e da gravidade das estenoses luminares, poderão esperar excelentes percentuais de sobrevida.

Resultados semelhantes, de estudos de pacientes não randomizados, relacionados à sobrevivência a longo prazo, foram obtidos em pacientes tratados clinicamente, publicados pelo Coronary Artery Surgery Study (CASS) ${ }^{5}$. Nesse estudo, $73 \%$ dos pacientes apresentavam angina estável e $48 \%$ haviam sofrido infarto do miocárdio previamente. A mortalidade observada após seis anos de seguimento aumentou proporcionalmente ao número de artérias comprometidas, sendo significativamente maior quando as estenoses eram proximais. Assim, nos portadores de estenoses arteriais nos três ramos principais, foi observada sobrevivência de $88 \%, 74 \%$ e $56 \%$ para lesões arteriais distais, e $77 \%, 58 \%$ e $40 \%$ para lesões arteriais proximais, respectivamente, para 1,2 e 3 vasos comprometidos. A fração de ejeção do ventrículo esquerdo determinou o maior impacto na sobrevida desses pacientes e foi considerada o maior fator de sobrevivência, independentemente do número de artérias comprometidas. Ainda, foi observado que o uso de drogas bloqueadoras adrenérgicas teve influência benéfica na prevenção de novo infarto e, também, na melhora dos sintomas.

Com o advento da cirurgia de revascularização miocárdica, foi observada uma acentuada diminuição de estudos eminentemente clínicos sobre o prognóstico da doença coronariana obstrutiva. Assim, vários fatores passaram a dificultar, ainda mais, a compreensão dos resultados entre si. Desta forma, diferenças na seleção de pacientes com quadros clínicos passíveis de interpretação ambígua e com vários graus de função ventricular contribuíram para dificultar as análises. Além disso, os objetivos dos estudos, principalmente voltados à sobrevida, têm dedicado pouca ênfase ao comportamento dos sintomas, surgimento de novos eventos, repetição dos angiogramas e terapêutica farmacológica, aqui destacados como aspectos relevantes a serem analisados.

Na expectativa da diminuição da incidência de morte, do infarto do miocárdio e do aumento da sobrevida, a cirur- 


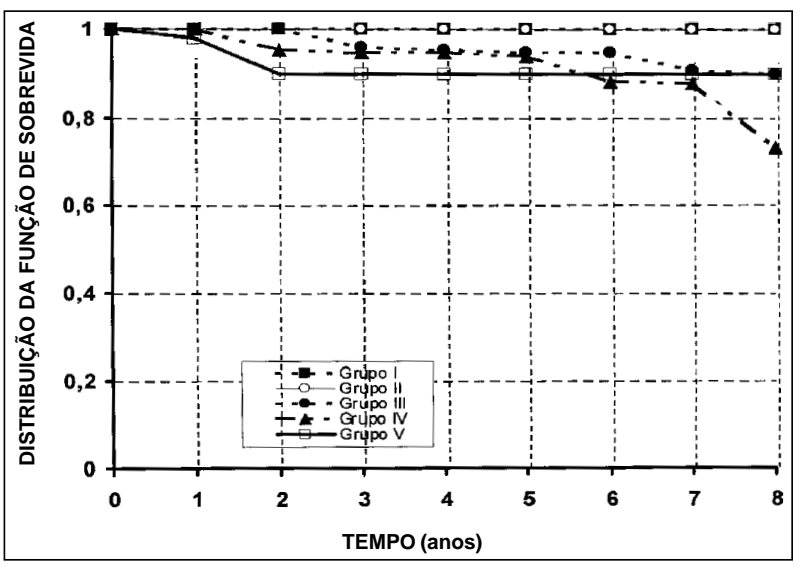

Fig. 1 - Sobrevida cumulativa de 8 anos para pacientes com lesões uni e multiarterial. Grupos I e II (uni e biarterial) sem mortalidade; grupos III, IV e V (triarterial, tronco equivalente e tronco da coronária esquerda) $1,5 \%$ mortalidade anual.

gia de revascularização miocárdica surgiu como opção promissora em determinados grupos de portadores de doença arterial coronariana sintomática. Entretanto, estudos realizados pelo CASS demonstraram que a cirurgia de revascularização do miocárdio não reduziu, significativamente, a incidência de morte ou a ocorrência de infarto do miocárdio em determinados subgrupos arteriais com angina estável e função ventricular preservada, quando comparados com pacientes que receberam tratamento clínico ${ }^{6}$. Dados obtidos na Duke Databank ${ }^{7}$ e do European Coronary Artery Surgery Study Group $^{8}$, somados a outros ensaios terapêuticos de revascularização cirúrgica do miocárdio ${ }^{9}$, mostraram resultados conclusivos, somente para determinadas condições clínicas, que a operação está associada a uma redução no risco de morte de pacientes com angina instável. O efeito benéfico da operação dependeu também, de dois outros fatores: o primeiro, relacionado ao importante comprometimento no tronco da artéria coronária esquerda e o segundo, ao importante comprometimento proximal dos três ramos principais, acompanhados de angina estável e disfunção miocárdica ${ }^{7-10}$. Em relação ao prognóstico em longo prazo, o efeito da operação de revascularização é complexo ${ }^{10,11}$. Sabe-se que no período imediatamente após a decisão para o tratamento cirúrgico, observa-se um aumento substancial de morte, quando comparado, no mesmo período, com pacientes que receberam indicação para tratamento clínico ${ }^{12}$. Os benefícios observados nos pacientes tratados com cirurgia, somente foram significantes e mais evidentes, a partir do $1^{\circ}$ ao $7^{\circ}$ ano de seguimento ${ }^{13}$. Após 11 anos de seguimento, a ocorrência de morte entre os dois grupos é equivalente.

Analisando os efeitos benéficos da operação, especificamente relacionados ao infarto não-fatal, observa-se que não diferem quando o tratamento é clínico. Entretanto, o risco precoce de infarto do miocárdio peri-operatório está aumentando no paciente que recebeu tratamento médico ${ }^{12}$. Esta desvantagem é anulada pelo aumento do risco da oclusão tardia do enxerto. Por outro lado, o surgimento da angioplastia transluminal coronariana, intervenção de relativa segurança e, aparentemente, efetivo meio de revascularizar o miocárdio, mudou drasticamente a tendência para seu tratamento ${ }^{14}$. Assim, apesar do uso generalizado da angioplastia transluminal coronariana, observam-se expressivos percentuais de infarto do miocárdio e subseqüente cirurgia de revascularização miocárdica, além do mais comum efeito adverso-a reestenose-que permanece inaceitavelmente elevada ${ }^{15}$.

Visto que a angioplastia transluminal coronariana não provou ser mais efetiva que cirurgia de revascularização miocárdica na redução da ocorrência de morte ou de infarto do miocárdio em subgrupos específicos, cabe inferir que seu potencial benefício é especificamente mensurado em termos de melhora funcional. Estudos iniciais e mais recentes do National Heart, Lung and Blood Institute (NHLBI) Registry ${ }^{16}$ demonstraram diferenças significativas entre sie, também, entre outras séries ${ }^{17-19}$ (tab. I). Diferenças que estão relacionadas, especificamente, aos objetivos do estudo, ou seja, percentual de infarto, de morte e alívio dos sintomas. As diferenças observadas estão repetidas também nos portadores de doença multiarterial ${ }^{16,19-22}$ (tab. II). Todavia, esses resultados devem ser analisados com cautela, pois há grande controvérsia no conceito de graduação da angina, de tipo de estenose, de sucesso do procedimento, de critérios para diagnosticar infarto e, até mesmo, na experiência dos operadores. Por outro lado, estudos recentes ${ }^{23}$, realizados em centros de maior experiência, não têm conseguido demonstrar melhora em tal condição.

Neste particular, estudo desenvolvido em nosso meio ${ }^{24}$ e realizado em única Instituição com critérios de seleção

\begin{tabular}{|c|c|c|c|c|c|}
\hline \multicolumn{6}{|c|}{$\begin{array}{c}\text { Tabela I - Resultados obtidos pela angioplastia nas estenoses } \\
\text { uniarteriais }\end{array}$} \\
\hline Estudo & $\begin{array}{l}\text { Pacientes } \\
\text { (N) }\end{array}$ & $\begin{array}{l}\text { Sucesso } \\
\text { ATC } \\
(\%)\end{array}$ & $\begin{array}{l}\text { IAM } \\
(\%)\end{array}$ & $\begin{array}{l}\mathrm{RM} \\
\text { Emerg. } \\
(\%)\end{array}$ & $\begin{array}{l}\text { Mortalidade } \\
\text { Hospitalar } \\
(\%)\end{array}$ \\
\hline${ }^{*}$ NHLBI ${ }^{16}$ & 863 & 69 & 5 & 25 & 1 \\
\hline${ }^{*}$ NHLBI ${ }^{16}$ & 839 & 89 & 4 & 5 & 0,2 \\
\hline Henderson et al ${ }^{17}$ & 412 & 85 & 6 & 2 & 0,5 \\
\hline Tazcu et al ${ }^{18}$ & 2677 & 93 & 3 & 5 & 0,3 \\
\hline Hubner et al ${ }^{19}$ & 4043 & ND & 2 & 2 & 0,4 \\
\hline \multicolumn{6}{|c|}{$\begin{array}{l}\text { * randomizado; ATC - angioplastia transluminal coronária; IAM- infarto } \\
\text { agudo do miocárdio; RM Emerg. - cirurgia de revascularização miocárdica } \\
\text { de emergência; ND - não disponível. }\end{array}$} \\
\hline
\end{tabular}

\begin{tabular}{|lccccc|}
\hline \multicolumn{7}{|c|}{ Tabela II - Resultados obtidos pela angioplastia nas estenoses } \\
multiarteriais
\end{tabular}


bastante rígidos, randomizou 214 pacientes com angina estável, lesão isolada na artéria descendente anterior e função ventricular normal. Esse estudo não apresentou diferenças entre as três opções terapêuticas disponíveis: angioplastia, cirurgia e tratamento clínico, quando analisou a ocorrência de infarto ou morte. Encontrou, todavia, maior ocorrência de eventos e subseqüentes reintervenções, via cateter-balão ou cirurgia de revascularização miocárdica, nos pacientes designados para tratamento através da angioplastia (fig. 2).

Estudo comparativo, realizado por Parisi e cols. ${ }^{25}$, comparou o tratamento clínico com intervenção através da angioplastia em portadores de doença uniarterial, onde se incluía também a artéria descendente anterior, e observou $3 \%$ de infarto do miocárdio no grupo clínico e $5 \%$ no grupo que recebeu angioplastia coronariana. Dados de relevância foram notados no índice de sucesso em $82 \%$, nova angioplastia em $9 \%$ e cirurgia de revascularização miocárdica em $7 \%$ e, ainda que não houvesse diferença significante na incidência de morte, o percentual de eventos nesse grupo específico de pacientes obriga-nos a profundas reflexões sobre os resultados.

Estudos mais recentes ${ }^{20-26}$, envolvendo pacientes com doença multiarterial, que receberam tratamento randomizado para angioplastia ou cirurgia, demonstraram a mesma tendência dos estudos anteriores, ou seja, índice elevado de eventos ou cirurgia emergencial nos pacientes randomizados para tratamento através da angioplastia (tab. III). Entretanto, portadores de doença coronariana multiarterial representam um grupo heterogêneo de condições anatômicas e clínicas em decorrência de diferentes locais de obstrução arterial e de vários graus de função ventricular. Estas condições aumentam as dificuldades de acesso à angioplastia ou de anastomoses coronarianas contribuindo assim, para tornar mais complexa a avaliação da eficácia de cada tratamento.

Por outro lado, as diferentes conceituações de doença coronariana multiarterial e de revascularização miocárdica, dita completa, contribuem para dificultar as comparações entre os estudos. Assim, duas questões devem ser coloca-

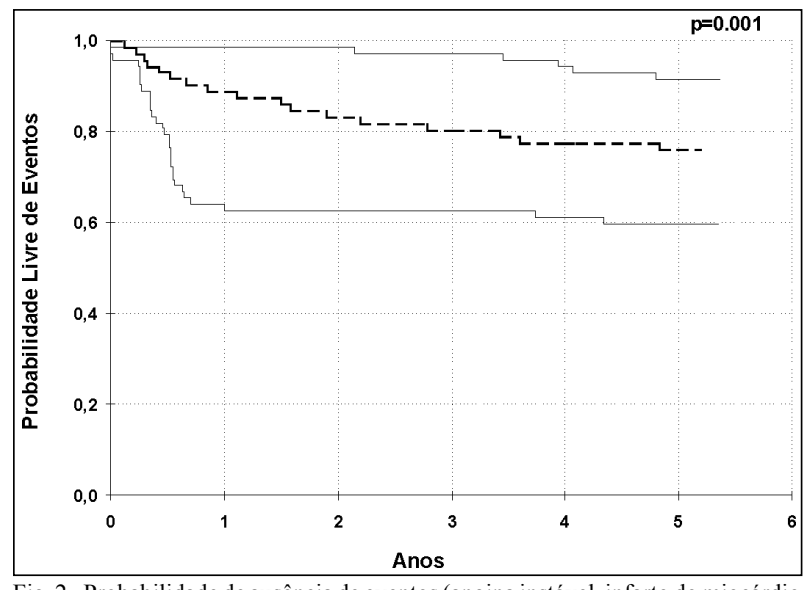

Fig. 2 - Probabilidade de ausência de eventos (angina instável, infarto do miocárdio e morte) para portadores de estenose isolada na artéria descendente anterior que receberam tratamento cirúrgico, clinico ou através da angioplastia.

\begin{tabular}{|c|c|c|c|c|c|c|}
\hline \multicolumn{7}{|c|}{ Tabela III - Angioplastia versus cirurgia - eventos na fase hospitalar } \\
\hline \multirow[t]{2}{*}{ Estudo } & \multicolumn{2}{|c|}{ Morte } & \multicolumn{2}{|c|}{ IAM } & \multicolumn{2}{|c|}{ RMEmerg } \\
\hline & $\overline{\mathrm{ATC}(\%)}$ & RM(\%) & $\overline{\mathrm{ATC}(\%)}$ & $\overline{R M}(\%)$ & ATC (\%) & $\overline{\mathrm{RM}(\%)}$ \\
\hline RITA $^{20}$ & 0,8 & 1,2 & 3,5 & 2,4 & 1 & 0 \\
\hline GABI $^{21}$ & 1,1 & 2,5 & 2,3 & 9,6 & 1,7 & 0 \\
\hline ERACI $^{23}$ & 1,5 & 4,6 & 6,3 & 6,2 & 0,9 & 1,5 \\
\hline EAST $^{24}$ & 1 & 1 & 3 & 10,3 & 1,7 & 0 \\
\hline BARI ${ }^{25}$ & 1,1 & 1,3 & 2,1 & 4,6 & 10,2 & 0,1 \\
\hline ARTS ${ }^{26}$ & 2,5 & 2,8 & 5,3 & 4 & 1,2 & 0,2 \\
\hline
\end{tabular}

das, objetivando o necessário entendimento dos resultados comparativos entre os tratamentos aplicados em portadores da doença coronariana multiarterial: identificar a proporção de pacientes com dois ou três vasos comprometidos e compará-los entre si e aos diferentes graus de função ventricular, pois esta condição, é fator relevante no prognóstico dos pacientes.

Finalmente, em muitos pacientes, especificamente naqueles com doença coronariana triarterial, é comum observar que, pelo menos, uma artéria coronária está cronicamente ocluída. Esta condição pode frustrar o médico intervencionista, diferentemente do cirurgião, a realizar uma revascularização miocárdica completa, reduzindo assim, significativamente, o índice de efetividade do procedimento.

Apesar de todas essas dificuldades, muitos estudos têm se dedicado a comparar os efeitos terapêuticos através da angioplastia ou da cirurgia de revascularização miocárdica em portadores de doença coronariana multiarterial.

Dentre esses, destacamos os estudos multicêntricos realizados na Europa ${ }^{26-28}$, os quais incluíram em suas amostras, $45 \%$ de pacientes com doença arterial coronariana unilateral e $43 \%$ de pacientes biarteriais ${ }^{26}$. Os outros dois estu$\operatorname{dos}^{27-28}$ alcançaram uma média de $71 \%$ de pacientes biarteriais e $29 \%$ triarteriais.

A presença de oclusão crônica da artéria coronária não foi fator de exclusão e o cirurgião ou hemodinamicista poderia intervir ou não nessa artéria. Desta forma, uma média de $85 \%$ dos pacientes triarteriais recebeu a revascularização cirúrgica completa, enquanto que somente $65 \%$ do grupo com angioplastia tiveram revascularização "via cateter" completa.

Durante o seguimento, não se observou diferença estatística em relação aos objetivos primários alcançados (infarto do miocárdio não fatal e morte cardíaca). Todavia, $41 \%$ dos pacientes submetidos a angioplastia necessitaram de nova revascularização miocárdica nesses grupos de estudos 27,28 .

Dois pequenos estudos unicêntricos realizados na Argentina ${ }^{29} \mathrm{e}$ Atlanta (EUA) ${ }^{30}$ incluíram em suas amostras um total de 519 pacientes onde $60 \%$ portavam doença biarterial. Um percentual relativamente aumentado de morte hospitalar ${ }^{31}$ deveu-se, provavelmente, a inclusão de pacientes com angina instável.

Igualmente aos anteriores, esses dois estudos mostraram elevado percentual de novas intervenções no grupo angioplastia e o uso de stents não estava disponível na época de sua realização. 
O Bypass Angioplasty Revascularization Investigation (BARI) recrutou pacientes em 16 hospitais americanos e em dois hospitais no Canadá, onde randomizou 915 pacientes para cirurgia e 914 para angioplastia. Com esse número de pacientes o BARI tornou-se o maior estudo comparativo e randomizado para doença coronariana multiarterial até o momento. Essa amostra randomizada foi extraída de um total de 25.200 pacientes com doença coronariana presuntiva, encaminhada para investigação. Dessa amostra, o BARI organizou um registro de 2.013 pacientes elegíveis para ambos os tratamentos, que não foram randomizados, por não obedecerem a critérios específicos do protocolo.

Assim, da amostra de 1.829 pacientes randomizados para angioplastia ou cirurgia, $58 \%$ eram portadores de estenoses biarterial e $42 \%$ de estenose triarterial, envolvimento da artéria descendente anterior presente em $1 / 3$ da amostra.

Em relação ao número de artérias coronárias através da cirurgia, observou-se que foi alcançada uma média de $3,1 \%$ de anastomoses coronárias por paciente e realizadas anastomoses com artéria mamaria em $82 \%$ dos pacientes.

A angioplastia foi realizada em múltiplas artérias em $70 \%$ dos pacientes e dilatações com sucesso, em pelo menos um vaso, em $88 \%$. Revascularização completa em todos os vasos propostos foi alcançada em $57 \%$.

Incidência de nova revascularização, quer através da cirurgia ou da angioplastia nos pacientes submetidos inicialmente a angioplastia, encontra-se na tabela IV. Assim, dos pacientes que receberam cirurgia de revascularização, $8 \%$ necessitavam de nova intervenção, predominantemente angioplastia, enquanto que, dos pacientes que receberam angioplastia, $54 \%$ necessitaram nova intervenção, em iguais proporções, cirurgia ou angioplastia. Nessa amostra observou-se que portadores de diabetes mellitus tratados através da angioplastia, tiveram pior prognóstico que aqueles que receberam tratamento cirúrgico. Ainda que esses resultados, iniciais tenham sido surpreendentes, torna-se razoável considerar, até que outras evidências conduzam a uma conclusão definitiva, que a opção cirúrgica para pacientes diabéticos tenha prioridade de escolha.

O Arterial Revascularization Therapies Study Group (ARTS) ${ }^{32}$ selecionou portadores de doença coronariana multiarterial estável que pudessem receber anastomoses

\begin{tabular}{|c|c|c|c|}
\hline \multicolumn{4}{|c|}{$\begin{array}{c}\text { Tabela IV - Pacientes Randomizados para angioplastia e que } \\
\text { receberam nova revascularização }\end{array}$} \\
\hline \multirow[t]{2}{*}{ Estudo } & \multirow{2}{*}{$\begin{array}{l}\text { Seguimento } \\
\quad \text { (anos) }\end{array}$} & \multicolumn{2}{|c|}{ Nova Revascularização } \\
\hline & & $\overline{\mathrm{ATC}(\%)}$ & $\mathrm{RM}(\%)$ \\
\hline RITA $^{26}$ & 2,5 & 18 & 19 \\
\hline $\mathrm{GABI}^{27}$ & 1,0 & 23 & 18 \\
\hline ERACI ${ }^{28}$ & 3,0 & 14 & 22 \\
\hline EAST 29 & 3,0 & 41 & 22 \\
\hline BARI $^{30}$ & 5,0 & 23 & 21 \\
\hline MASS-II ${ }^{32}$ & 1,0 & 11 & 6 \\
\hline $\mathrm{CABRI}^{33}$ & 1,0 & 20 & 15 \\
\hline
\end{tabular}

coronarianas ou angioplastia em número equivalente para ambos procedimentos.

Assim, foram randomizados 1.205 pacientes para tratamentos cirúrgico ou através da angioplastia. Desses pacientes, $68 \%$ eram portadores de doença biarterial e $32 \%$ de doença triarterial.

Os objetivos principais da análise dos resultados incluíram a comparação combinada da incidência de morte e infarto do miocárdio. Curiosamente, foi incluído nesta análise, o acidente vascular cerebral como evento combinado para os dois grupos terapêuticos estudados.

Desta amostra randomizada, $99 \%$ dos pacientes designados para angioplastia e $96 \%$ dos designados para cirurgia receberam seus respectivos tratamentos.

A implantação de stents foi alcançada em $89 \%$ dos pacientes, enquanto que tratamento com uso de balão isoladamente, completou os $11 \%$ dos pacientes restantes.

Assim, após um ano de seguimento, não se observaram diferenças significativas entre os dois grupos estudados, em relação à incidência de eventos combinados, definidos como objetivos principais.

Entretanto, 16,8\% (102 pacientes) do grupo angioplastia necessitaram de uma $2^{\mathrm{a}}$ revascularização, enquanto que somente $3,5 \%$ (21 pacientes) do cirúrgico receberam uma nova intervenção.

O Medicine, Angioplasty and Surgery Study (MASSII) ${ }^{33}$ foi realizado em umúnico hospital - Instituto do Coração em São Paulo - utilizando um registro de 20.769 pacientes, randomizou 611 portadores de doença coronariana multiarterial, angina estável e função ventricular preservada. Desses pacientes, $42 \%$ eram portadores de estenoses biarteriais e $58 \%$ triarteriais. A randomização foi planejada para grupar os pacientes nos três principais métodos terapêuticos disponíveis; assim tornou-se o único trabalho randomizado que contemplou o "braço" clinico para comparação terapêutica e foi o que reuniu o maior percentual de pacientes triarteriais.

Os objetivos principais da análise dos resultados incluíram a comparação combinada da incidência de infarto do miocárdio, morte e angina instável que requeresse intervenção em qualquer grupo.

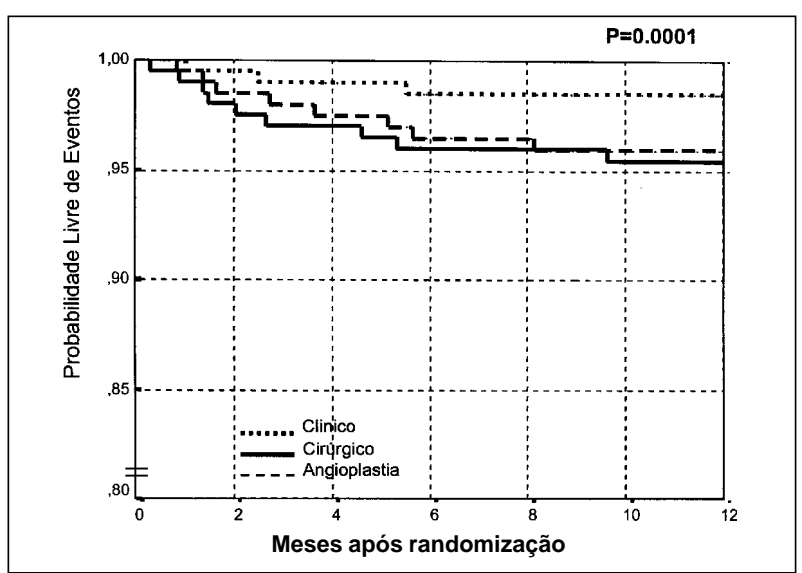

Fig. 3 - Probabilidade de ausência de eventos angina instável, infarto do miocárdio e morte. 
Desta amostra randomizada, $97 \%$ dos pacientes selecionados para cirurgia receberam o tratamento, $94 \%$ daqueles randomizados para angioplastia tiveram o procedimento realizado. O número de anastomoses por paciente alcançou $3,3 \pm 0,8$ e o número médio de estenoses tratadas por paciente atingiu $2,1 \pm 0,7$. O sucesso imediato do procedimento via cateter alcançou $92 \%$ e $72 \%$ dos pacientes, receberam pelo menos um stent. No grupo cirúrgico, o uso de artéria mamária como anastomose alcançou $92 \%$ dos pacientes revascularizados.

Durante o seguimento de um ano, 25 pacientes do grupo angioplastia sofreram nova revascularização, 16 do grupo clinico receberam uma intervenção e um do grupo cirúrgico foi submetido a nova revascularização. A probabilidade livre de eventos combinados encontra-se na figura 3.

Assim, após análise criteriosa desses estudos, é importante considerar que os pacientes incluídos nos estudos randomizados representam uma pequena parcela dentro do universo de pacientes que tem alguma indicação para procedimentos, seja para cirurgia ou angioplastia. De fato, em todos os estudos randomizados, observamos que pelo menos $70 \%$ dos pacientes elegíveis para qualquer um destes tratamentos, foram excluídos por critérios rigorosos da randomização e, portanto, não foram contemplados para ter sua evolução estudada.

Além disso, considerando-se a variabilidade anatômica das artérias coronárias, o grau de importância de cada uma, a área miocárdica sob risco, as artérias cronicamente ocluídas, as quais dificultam o procedimento via cateter, constatamos também que, na maioria dos estudos, a composição dos grupos arteriais reunia pelo menos $60 \%$ dos pacientes com estenose biarterial, aceito universalmente, como pacientes de baixo risco e os $40 \%$ restantes, com envolvimento nas três artérias.

Por outro lado, a análise da quantidade média de procedimentos por um ou outro método revelou que existe grande diferença na quantidade de artérias tratadas entre intervenções. De maneira geral e com exceção do estudo ARTS, a intervenção cirúrgica alcançou, aproximadamente, em $50 \%$ a mais, onúmero de artérias revascularizadas que a angioplastia.
Ora, se, em termos de sobrevivência, os resultados de ambos procedimentos, em todos os trabalhos, são superponíveis, é razoável refletir sobre quantas e quais artérias devam ser tratadas, por qualquer um dos métodos.

De qualquer maneira, as lições que esses estudos têm revelado estão sendo incorporadas à prática clínica e os avanços observados nestas três modalidades terapêuticas têm revelado melhora substancial em todas as abordagens da doença arterial coronariana crônica estável.

Em relação ao tratamento clínico, o uso de betabloqueadores, de drogas antiplaquetárias e novas gerações de estatinas podem, provavelmente, melhorar os resultados não somente dos pacientes tratados, clinicamente, como naqueles que recebem outra forma de revascularização. Quanto à revascularização percutânea, a reestenose representa um papel importante nos resultados de todos esses estudos, aqui analisados, e é objeto de intensa investigação.

Esses estudos têm avaliado, especificamente, as interações entre as plaquetas, trombos e parede do vaso; a gênese, a evolução da placa aterosclerótica e suas possíveis implicações com técnicas de biologia molecular. Além disso, novos avanços na aquisição e uso de recursos mecânicos e farmacológicos em desenvolvimento podem, provavelmente, diminuir a freqüência e o grau de reestenose e influenciar nos resultados das futuras comparações entre estas três modalidades terapêuticas da doença coronariana obstrutiva crônica.

Finalmente, a curva de aprendizado da cirurgia de revascularização miocárdica considerada, relativamente estática, pode ser dinamizada com a introdução de técnicas cirúrgicas, minimamente invasivas, como anastomoses e novas formas de proteção miocárdica. Estes importantes avanços, em todas as formas de tratamento da doença coronariana estimularão futuras comparações de estudos randomizados.

Odesafio maioré determinar qual é o mais efetivo método de tratamento da doença coronariana para um paciente isoladamente, quando estão em jogo as diversidades das síndromes isquêmicas, história natural da doença, anatomia coronariana, função ventricular, objetivo desejado e, finalmente, a preferência do paciente.

\section{Referências}

1. Lim JI, Proudfit WI, Sones FM Jr. Left main coronary arterial obstruction: longterm follow-up of 141 nom-surgical cases. Am J Cardiol 1975; 36: 131-5.

2. Webster JS, Moberg C. Natural history severe proximal coronary artery disease as documented by coronary cineangiography. Am J Cardiol 1974; 33: 195-200.

3. Harris PJ, Phil D, Harrell FE, lee HL, Bechar VS, Rosati RA. Survival in medically treated coronary artery disease. Circulation 1979; 60: 1259-69.

4. Hueb W, Bellotti G, Ramires JAF, Da Luz PL, Pilleggi F. Two-to eight-year survival rates in patients who refuse coronary artery bypass grafting. Am J Cardiol 1989; 63: 155-9.

5. Mock MB, Ringqvist I, Fisher LD. Survival of medically treated patients in the Coronary Artery Surgery Study (CASS) Registry. Circulation 1982; 66: 562-8.

6. CASS Principal Investigators and their Associates. Coronary Artery Surgery Study (CASS): a randomized trial of coronary artery bypass surgery. Circulation 1983; 68: 939-50.

7. Callif RM, Harrell FE Jr, Lee KL, et al. The evolution of medical and surgical therapy for coronary artery disease: a 15-year-perspective. JAMA 1989; 261: 2077-88.

8. European Coronary Surgery Study Group. Long-term result of prospective randomized study of coronary artery bypass surgery in stable angina pectoris. Lancet 1982; 11: 1173-81.

9. Murphy M, Hultgreen H, Detre K, Thomsen J, Takaro T and participants of the Veterans Administration Cooperative Study. Treatment of chronic stable angina: a preliminary report of survival data of the Randomized Veterans Administration Cooperative Study. N Engl J Med 1977; 297: 621-9.

10. Kirklin JW, Akins CW, Blackstone EH, et al. ACC/AHA guidelines and indications for coronary artery bypass graft surgery. Circulation 1991; 83: 1125-34.

11. VA Coronary Artery Bypass Surgery Co-Op Study Group. Eighteen-year follow-up in the Veterans Affairs Cooperative Study of coronary artery bypass surgery for stable angina. Circulation 1992; 86: 121-7.

12. Force T, Hibberd P, Weeks G, et al. Perioperative myocardial infarction after coro- 
nary artery surgery: clinical significance and approach to risk stratification. Circulation 1990; 82: 903-11.

13. Vernauskas E, European Coronary Surgery Study Group. Twelve-year followup of survival in the randomized European coronary study. N Engl J Med 1988; 319: 332-9.

14. Pepine CJ, Hill JA, Lambert CR. Therapeutic cardiac catheterization. Part 1. Mod Concepts Cardiovasc Dis 1990; 59: 55-60.

15. Popma JJ, CallifRM, Topol EJ. Clinical trials of restenosis after coronary angioplasty. Circulation 1991; 84: 1426-36.

16. Dentre K, Holubkov R, Kelsey S, et al. Percutaneous transluminal coronary angioplasty in 1985-1986 and 1977-1981. N Engl J Med 1988; 318: 263-71.

17. Henderson RA, Karani S, Dritsas A, Sowton E. Long-term results of coronary angioplasty for single vessel, proximal, left anterior descending disease. Eur Heart J 1991; 12: 642-9.

18. Tuzcu EM, Simpfendorfer C, Badhwar K, et al. Determinants of primary success in elective percutaneous trasluminal coronary angioplasty for significant narrowing of a major coronary artery. Am J Cardiol 1988; 62: 873-80.

19. Hubner P. Cardiac interventional procedure in the United Kingdom in 1989. Br Heart J 1991; 66: 469-58.

20. Vandormael MG, Chaitman BR, Ischinger T, et al. Immediate and short-term benefit of multilesion coronary angioplasty: influence of degree of revascularization. J Am Coll Cardiol 1985; 6: 983-92.

21. Bell MR, Bailey KR, Reeder GS, et al. Percutaneous transluminal angioplasty in patients with multivessel coronary disease: how important is complete revascularization for cardiac event-free survival? J Am Coll Cardiol 1990; 16: 553-61.

22. Deligomel U, Vandormael MG, Kern MJ, et al. Coronary angioplasty: a therapeutic option for symptomatic patients with two and three vessel coronary disease. J Am Coll Cardiol 1988; 1173-9.

23. Topol EJ, Leya F, Pinkerton CA, et al. A Comparison of directional atherectomy with coronary angioplasty in patients with coronary artery disease. NEng J Med 1993; 329: 221-7.
24. Hueb WA, Bellotti G, Oliveira AS, et al. The medicine angioplasty or surgery Study (MASS): a prospective, randomized trial of medical therapy, balloon angioplasty or baypass surgery for single proximal left anterior descending artery stenosis. J Am Coll Cardiol 1995; 26: 1600-5.

25. Parisi AF, Folland ED, Hartinger P. A comparison of angioplasty with therapy in the treatment of single vessel coronary artery disease. N Engl J Med 1992; 326: $10-6$.

26. RITA Trial Participants - Coronary angioplasty versus coronary artery surgery: the randomized intervention treatment of angina (RITA) Trial 1993; 341: 573-8.

27. Hamm CW, Reimers J, Ischinger T, Rupprecht HJ, Berger J, Bleife, randomized study of coronary angioplasty compared with bypass surgery patients with symptomatic multivessel coronary disease. N Engl J Med 19; 1037-43.

28. First-year results of CABRI (Coronary Angioplasty Versus Bypass Revascularization Investigation). CABRI Trial Participants. Lancet 1995; 346: 1179-84.

29. Rodriguez A, Boullon F, Perez-Balino, Paviotti C, Liprandi MI, Palacios IF. Argentine randomized trial of percutaneous transluminal coronary angioplasty versus coronary artery bypass surgery in multivessel disease (ERACI): -in-hospital results and 1-year follow-up. ERACI Group. J Am Coll. Cardiol 1993; 22: 1060-67.

30. King SB III, Lembo JN, Weintraub SW, et al. A randomized trial comparing coronary angioplasty with coronary bypass surgery: emory Angioplasty versus Surgery Trial. N Engl J Med 1994; 331: 1044-50.

31. The Bypass Angioplasty Revascularization Investigation (BARI) Investigators. Comparison of coronary bypass surgery with angioplasty in patients with multivessel disease. N Engl J Med 1996; 335: 217-25.

32. Serruys PW, Unger F, Souza JE, et al, For The Arterial Revascularization Therapies Study Groups (ARTS). Comparison of coronary-artery bypass surgery and stentting for the treatment of multivessel disease. N Engl J Med 2001; 344: 15 : 1117-24.

33. The Medicine, Angioplasty or Surgery Study (MASS-II). A randomized controlled clinical trial of three therapeutic strategies for multivessel coronary artery disease: one-year results (Abstract). Eur Heart J 2001; 22 (Suppl.). 